\title{
Ley departamental para la otorgación de tarjetas de operaciones del transporte interprovincial
}

\author{
Departmental law for the granting of interprovincial transportation operations cards
}

Lei departamental de concessão de cartões de operações de transporte interprovincial

Artículo recibido en abril 2019

Arbitrado en mayo 2019

Publicado en julio 2019

\section{Flavio Raúl Quijandria Rojas}

flaquijandria@hotmail.com

ORCID: 0000-0002-6472-357X

Universidad Privada Domingo Savio, Bolivia

El transporte es un derecho que es adquirido por la población, a pesar de los vacíos legislativos existente. La investigación tuvo como objetivo general proponer una ley para la regulación de las tarjetas de operación del transporte terrestre interprovincial de pasajeros, la metodología estuvo bajo un enfoque mixto cualitativo y cuantitativo con un diseño documental, de campo. Se usó el modelo empírico con los métodos inductivos, deductivos, entre otros. La población y muestra, fue realizada a los pasajeros que habitualmente hacen viajes a las provincias y la muestra se conformó por 200 personas seleccionada de manera aleatoria y al azar. La técnica e instrumento que se usó para la recolección de los datos fue la encuesta y el cuestionario. Como resultado se obtuvo la ausencia de una ley en el Departamento, por lo que fue necesario contar con una normativa para un control sobre los servicios que dan los transportistas.

Palabras clave: Transporte; tarjetas de operaciones; derecho comparado; ley departamental, servicio público

\begin{abstract}
Transportation is a right that is acquired by the population, despite the existing legislative gaps. The general objective of the research was to propose a law for the regulation of interprovincial passenger land transport operation cards; the methodology was under a mixed qualitative and quantitative approach with a documentary, field design. The empirical model was used with inductive and deductive methods, among others. The population and sample were made to passengers who habitually make trips to the provinces and the sample was made up of 200 people selected randomly and at random. The technique and instrument used to collect the data was the survey and the questionnaire. As a result, the absence of a law in the Department was obtained, so it was necessary to have a regulation to control the services provided by carriers.
\end{abstract}

Key words: Transportation; trading cards; comparative law; departmental law, public service

RESUMO

O transporte é um direito adquirido pela população, apesar das lacunas legislativas existentes. O objetivo geral da pesquisa foi propor uma lei para a regulamentação dos cartões de operação de transporte terrestre de passageiros interprovinciais, a metodologia obedeceu a uma abordagem qualitativa e quantitativa mista com um desenho de campo documental. O modelo empírico foi utilizado com métodos indutivos e dedutivos, entre outros. A população e a amostra foram constituídas por passageiros que habitualmente fazem viagens às províncias e a amostra foi constituída por 200 pessoas selecionadas de forma aleatória e aleatória. A técnica e o instrumento utilizados para a coleta de dados foram a votação e o questionário. Com isso, obteve-se a inexistência de uma lei no Departamento, sendo necessária uma regulamentação para controlar os serviços prestados pelas operadoras.

Palavras-chave: Transporte; cartões comerciais; lei comparativa; lei departamental, serviço público 


\section{INTRODUCCIÓN}

El proceso de evolución continúa en la manera que incita al hombre a buscar medios y modos para adaptarse y satisfacer sus necesidades de poder transportarse de un lugar a otro que ayudan considerablemente en el desarrollo de la civilización y está al servicio del interés público y como al movimiento de todo pasajero del servicio del transporte público que hace uso.

El transporte constituye un derecho adquirido por la población donde el Estado y los gobiernos Autónomos Departamentales se conviertan en garantes de este servicio para contribuir en la calidad de vida de los habitantes de las ciudades. Y siendo la movilización una de las necesidades básicas de la población donde el Estado es garante por cubrir esta necesidad, se hace necesario que el Gobierno autónomo Departamental de Potosí contribuyen en la mejorar donde la calidad del servicio de transporte, entendiendo a la formulación de políticas, como el proceso mediante el cual el Estado planifica y forma acciones con la finalidad de cubrir las necesidades básicas de la sociedad. En el caso del transporte tal como lo señala.

Para Ocaña (2003), "el objetivo de una política de transporte no es simplemente de movilizar a los usuarios sino aumentar, de manera global el bienestar de los habitantes" ( $p$ 87). De esta forma, en el Departamento de Potosí se evidencia la presencia la falta de control al transporte interprovincial terrestre organizado, que en muchos de los casos es formal o informal y entre ellos se destaca el servicio prestado por los vehículos, minis, buses, expresos que realizan viajes interprovincial ,se evidencia una serie de falencias, falta de calidad de atención a los pasajeros, el poco mantenimiento que se hace los vehículos, todo por la falta de control y fiscalización al transporte público. Se considera que existen vacíos legales que puedan normar esta actividad del transporte, como la falta de regulación de los vehículos a partir de la otorgación de tarjetas de operaciones interprovinciales.

A su vez, el transporte es considerado como un sistema que efectúa el desplazamiento de usuarios del servicio vehicular, el cual, debe y tiene que ejercer una calidad y la fiscalización a los medios de transporte cuyo objetivo será el de buscar la seguridad del pasajero en el momento de trasladarse de un lugar a otro, de las provincias.

Por ello, es que se detectó durante el diagnóstico de la investigación, que no existe una norma que regule el servicio de transporte interprovincial, se observa una falencia en los recursos técnicos de control y la fiscalización. Si bien. en la Gobernación de Potosí, cuenta con una unidad de transporte que es subordinada a otra, está se la debe jerarquizar a partir de la elaboración de una Ley Departamental, para que tenga la autonomía y decisiones propias en el campo del transporte. 
El Transporte Interprovincial del Departamento de Potosí se encuentra en vacío legal de problemas de transporte no existiendo una normativa correspondiente en el Departamento, además existen importantes vías de comunicación que entrelazan el departamento en él se reflejan los problemas de control que no se efectúa por la autoridad competente, con el propósito de mejorar un servicio de calidad al transporte interprovincial de pasajero.

Conforme a los estudios realizados sobre el Transporte Interprovincial como; un fenómeno complejo, con características especiales, que incluso dependen del enfoque con que se analice se trata de una problemática que existe en todo los países principalmente respecto del mal funcionamiento del orden del transporte desde un punto de vista económico, el transporte conecta los sectores productivos a la vez con los centros de consumo, de esta forma todo lo que se fabrica es puede ser trasladado con atención a dicho lugares donde se consume, si bien en su parte facilita dicho desplazamiento de las personas y la mercaderías para dicho el desplazamiento sin embargo no es un servicio de buena calidad.

Por lo tanto, Valero (1970), considera que "el transporte, como actividad económica, productiva y de renta, debe considerar el transporte individual como actividad económica y objeto de consumo, por ejemplo para el transporte entre lugares de residencia y de trabajo o para el consumo de ocio" (p. 65). En todo caso, no se adecua a las necesidades del usuario por la debida calidad del, servicios que se pretende dar.

Seguidamente, la Constitución Política del Estado vigente desde 2009, asigna como competencia exclusiva, el transporte interprovincial. Las competencias que se refieren a la elaboración de Planes de Ordenamiento territorial, diseño, construcción y administración de carreteras de la red departamental, y el transporte interprovincial por todos los medios de transporte.

Es por ello, que el Gobierno Departamental debe asumir las facultades legislativa, reglamentaria y ejecutiva, y la Ley Marco de Autonomías amplía esta competencia, como determina la CPE para poder normar el transporte también emplear políticas a los referente al transporte interprovincial una nueva filosofía de fiscalización considerándola una herramienta para promover la formalización del transporte terrestre, así como medio impulsor del desarrollo y progreso y de esta forma permitir accesibilidad y comunicación en las provincias dentro una cultura de paz seguridad de los pasajeros y como medio impulsor al desarrollo.

A su vez, la ley General del Transporte N¹65 establece que las diferentes modalidades de transporte están regidas por la autoridad competente en el ámbito de su jurisdicción, aclarando en el inciso b) que la autoridad competente del nivel departamental, es el representante del Órgano Ejecutivo del nivel departamental destinado a emitir políticas, planificar, regular, fiscalizar y administrar la ejecución, 
gestión, operación y centros del Sistema de Transporte, además de aprobar planes y proyectos relativos al transporte. Las competencias de los municipios, son solamente para el radio urbano. En cuanto el vehículo sale a otro municipio o a otra provincia, ya es competencia de la Gobernación.

Así mismo el Decreto de Santa Cruz y Cochabamba la Paz y de Colombia sientan bases para normar y regulan y fiscalizar el control de las tarjetas de operaciones para mejorar calidad del transporte y poder resolver los problemas que continuamente existen a falta de una normativa, para hacer uso de un debido control a todo medio del transporte y poder cortar con un control minucioso de los vehículos que prestan este servicio, por lo que se evidencia la necesidad de implementar una propuesta de ley para la regulación de las tarjetas de operación del transporten, siendo éste, el objetivo central de la investigación. Logrando de esta manera, como aporte, mejorar el servicio y la seguridad de todos los pasajeros del departamento.

\section{Marco histórico}

\section{Trasporte Público}

En el ámbito terrestre, la aparición de la rueda llevo a la existencia de los carros primitivos, que según parece, surgieron cuando, para favorecer el deslizamiento, se sujetaron un par de discos de un tronco al objeto que era necesario transporta. Estos carros aparecieron en Mesopotamia sobre el 3500 a. C. y se difundieron en Creta y Egipto alrededor del año 2000 y 1600 a. c respectivamente. Los egipcios fueron los que lograron tener los mejores carros del mundo porque su ligereza le confería el máximo rendimiento de la velocidad. Transporte por medio de los carros, tambiénse utilizaron para el traslado de personas, que resultaron un medio de locomoción apropiado que avanza por tierra y la eficiencia del transporte.

Según Hawks (1946) manifiesta que:

Los carros debieron existir desde muy antiguos, porque se mencionan varias veces en las fuentes de los grandes libros mitológicos y religiosos, delos que han nutrido a los estudios antigua, como la Biblia o la "La Ilíada". En Europa, estos vehículos se propagaron entre 1000 y el 500 a.C. Como ha ocurrido con la frecuencia dela historia, los primeros vehículos con ruedas se emplearon más para la guerra que para el comercio y su introducción en los campos de batalla genero un gran pánico a un enemigo que era lento y poco móvil. (p. 55). 
De esta manera, el transporte es un derecho que es adquirido por la población que tiene como desplazarse de un lugar a otro como entre provincias para que puedan desarrollar sus propias necesidades y es un derecho que ha adquirido la población que tiene sus propios elementos y características y la necesidad de los, pasajeros también es la obligación del Gobierno Autónomo de regularlo mediante normas y de forma que se respete este derecho y se establezca obligaciones tanto para los operadores de transporte de pasajeros.

Por lo tanto, Osorio (2002), señala que: "cuando el transporte se refiere a personas, la obligación del transportista consiste en facilitar al pasajero el recorrido de un itinerario previamente determinado" (p. 345).

A su vez, Lane, y Powell (1976), plantea que existen dos clases de transporte:

...primero, el de productos manufacturados o materias primas a, o desde el lugar de venta o producción. Segundo, el delas personas que necesitan recorrer la distancia que separa físicamente los lugares donde deben desarrollar las actividades que realizan durante el día. (p. 45).

Es entendido por servicio público, a las actividades, entidades u órganos públicos o privados con personalidad jurídica creados por Constitución o por ley, para dar satisfacción en forma regular y continua a cierta categoría de necesidades de interés general, bien en forma directa, mediante concesionario o a través de cualquier otro medio legal con sujeción a un régimen de Derecho Público o Privado, según corresponda.

\section{Servicio público}

Para Fernández (2002), Servicio público es:

Toda actividad técnica destinada a satisfacer una necesidad de carácter general, cuyo cumplimiento uniforme y continuo deba ser permanentemente asegurado, reglado y controlado por los gobernantes, con sujeción a un mutable régimen jurídico exorbitante del derecho privado, ya por medio de la Administración Pública, bien mediante particulares facultados para ello por autoridad competente, en beneficio indiscriminado de toda persona. (p. 121).

El transporte regular se ubica dentro de la clasificación del servicio público propio y privado por estar expresamente establecido en la ley como tal y, en consecuencia atribuido a la administración pública, quien lo pueda prestar directamente o de manera indirecta, por medios particulares como se indican en la ley de transporte: Por otra parte la Ley general de Transporte $N^{\circ} 165$ como objeto de establecer: 
Artículo 75.- (Transporte público). El servicio de transporte público, se define como aquel que tiene el propósito general de satisfacer la necesidad que originan prestaciones dirigidas a las usuarias y los usuarios individualmenteo en su conjunto, que son de interés público y sirvan al bien común;comprenden las unidades de transportes en que las usuarias y los usuarios no son los propietarios de los mismo, siendo prestados comercialmente por terceros y pueden ser suministrados tanto por instituciones públicas y privadas

\section{Clases de trasporte}

Existen diversas clases de transporte que de acuerdo a sus funciones, el servicio público de transporte tiene por objeto satisfacer necesidades individuales de importancia colectiva, en una demanda derivada al uso del servicio en cualquier transporte no es un fin en sí mismo es general, por el consumidor del mismo que se desplaza para satisfacer diversas necesidades se clasifican en: Transporte Marítimo, por Carretera, Ferroviaria, y Aéreo.

\section{Calidad de transporte}

El término calidad ha sido ampliamente utilizado en distintos sectores de la seguridad $y$, dentro de cada sector en distintos niveles. El concepto de calidad ha sido considerado, en sus orígenes como un término general e indefinido y de interés general como el servicio y transporte en la razón de brindar un buen servicio que se prestar en la sociedad consumidores y los usuarios. Por lo tanto, Núñez (2001) que "la calidad tiene diferentes ocupaciones según el área donde se aplica en atribuir de un producto o servicio que se refleja su habilidad para satisfacer las necesidades del consumidor de las expectativas del servicio que puedan brindar la calidad a los servicios" (p. 143).

Por lo tanto, para llegar a niveles de atención con calidad al usuario debe existir unaarmonía total entre la calidad de todos los servicios generales, pues tomando en cuenta la calidad se piensa inmediatamente en cliente usuario (pasajero) que implica la necesidad del usuario o del cliente del servicio, para cubrir las expectativas a la satisfacción del servicio.

Así que, para Lewis y Booms.(1983):

La calidad del servicio es la caracterización entre las expectativas y la percepción del resultado de una comparación, entre lo que se consideran debe ser el servicio ofrecido por empresas y su persecución del rendimientode los propietarios del servicio, de esta forma, se establece que la calidad de servicio la expectativas y la persecución del resultados, es 
decir de una evaluación en cual el usuario compara la experiencia que a ha tenido con elservicio, busca responder a las expectativas de los clientes ( $p$. 259).

\section{Tarjetas de operaciones}

Según el Decreto que reglamenta la prestación del servicio público de pasajeros, da una definición general sobre las tarjetas. La Tarjeta de Operación es el documento único que autoriza a un vehículo automotor para prestar el servicio público de transporte de pasajeros bajo laresponsabilidad de una empresa, de acuerdo con los autorizados y/o registrados". (Decreto Supremo No 659, 6 de octubre de 2010, Bolivia).

Llegando a entender que la tarjeta de operaciones es aquel documento otorgado por autoridad competente para que el servicio público de transporte de pasajeros este sujeto a las disposiciones en procura de un control y fiscalizar servicio eficiente, seguro y cómodo; en el transporte de pasajeros.

\section{Legislación Nacional de transporte}

El Estado Plurinacional de Bolivia, se fundamenta en leyes formales, estas leyes formales constituyen el instrumento jurídico que se emplea para establecer las estructuras organizacionales y funciones administrativas, la Constitución Política del Estado establece que el ejecutivo, contara con los ministerios de competencias y atribuciones y los Gobiernos Departamentales tienen las atribuciones de legislar, en base a lo expuesto y partiendo de nuestra Constitución Política de Bolivia cabe mencionar la legislación las leyes y normas que regula lo relativo al sector del transporte a la solución y la regulación.

El marco normativo en el cual se desarrollan estas acciones tiene el siguiente fundamento legal: Constitución Política del Estado Plurinacional de Bolivia; Ley Marco de Autonomías y Descentralización, y Ley General del Transporte.

\section{MÉTODO}

El estudio se desarrolló bajo el enfoque mixto (cuantitativo como cualitativo), para desarrollar y determinar la fuerza entre las variables especificas con la finalidad de realizar los resultados a través de las muestras para hacer la diferencia y la población de cual procede la muestra. La investigación consistió fundamentalmente en la aplicación de una metodología basada en los métodos empíricos como la encuesta, la entrevista y el análisis documental para medir las dimensiones del conocimiento del estudio sobre los problemas de la regulación del transporte propiamente.

Tal y cual, lo señala Rubio y Varas (1995): 
Describir y explicar los hechos sociales desde sus manifestaciones externas. Con lo que desde esta vía se aborda el nivel de los hechos o fácticos del contenido dela realidad social en el que se sitúa todos los fenómenos sociales manifiestos que son perceptibles y registrados a partir de la observación del investigador (p. 229).

A su vez, la forma en que se aplicó el enfoque cuantitativo en la investigación fue la tabulación de las encuestas, que permitió cuantificar las respuestas emitidas por los encuestados pasajeros acerca de una ley de las tarjetas operaciones.

En cambio, el enfoque cualitativo de investigación es aquél, según Taylor y Bogan (1986), "que produce datos descriptivos, las palabras propias de las personas, en forma hablada o escrita, y laconducta observable" (p. 33).

El diseño de la investigación fue documental, de campo, ya que se aplicó la encuesta, y la entrevista, en forma, directa, del comportamiento y actitud de las personas en su condición de pasajeros y autoridades en el departamento de Potosí, en cuanto a la falta de una ley departamental, así mismo, dado que no existe ni un estudio o antecedentes en el transporte en el Departamento de Potosí sobre la problemática planteada, para un mejoramiento y la calidad del transporte. Planteado

Sin embargo, al desarrollarse bajo un modelo empírico se usó los métodos de análisis y síntesis, la cual, permitió efectuar un estudio minucioso y a la vez detallar todos los aspectos y elementos teóricos necesarios que engloba el objeto de estudio, asimismo permitió observar el problema tal como se presenta en la realidad. La síntesis, posibilitó sistematizar los elementos más importantes y necesarios que facilitó un pleno conocimiento de la realidad del problema con la población de estudio. El inductivo, ya que, se obtuvo información acerca de las expectativas de los pasajeros y que sirvieron de fundamento de análisis de resultados y redacción de las conclusiones de la investigación, es decir de lo general a lo particular. El deductivo, porque partió de los conceptos y teorías desarrollados en el marco teórico conceptual y fueron aplicadas al tema de estudio, y la doctrina en Transporte y los mecanicismos para una solución pacífica del buen servicio y la necesidad de transporte de un lugara otro. Histórico-Lógico, ya que, permitió ordenar los hechos ocurridos cronológicamente en una secuencia ordenada; es decir, en la recopilación de los antecedentes sobre tarjetas deoperaciones del transporte, desde el surgimiento hasta el desarrollo, a través del tiempo que, a partir de ello se determinaron y organizaron los fundamentos históricos del problema en estudio.

En cuanto, a la población y muestra que se usó en la investigación, fue realizada a los pasajeros que habitualmente hacen viajes a las provincias, a quienes se les aplicó la encuesta, es decir, los conductores, pasajeros. A su vez, la muestra fue aleatoria al azar (cada elemento de la población tiene la misma oportunidad de participar) y se 
obtuvo con la fórmula estadística para poblaciones finitas, cuyo resultado de la aplicación se dio a la muestra.

Por lo tanto, Hernández-Sampieri, Fernández-Collado, y Baptista-Lucio (2006), señalan al respecto que: "La selección de muestra para cada población o estrato, la estratificación aumenta la precisión de muestra implica el uso deliberativo de diferentes tamaños de muestra para cada estrato a fin de lograr reducir la varianza". (p. 134).

Las técnicas e instrumentos que se usaron para la recolección de los datos de la investigación fueron, la encuesta, entrevista, y la revisión documental, y el instrumento usado fue el cuestionario.

La encuesta constituyó una de las herramientas más importantes, para alcanzar los objetivos de la investigación y fue realizada teniendo en cuenta ciertos criterios estructurales en su construcción y el apropiado manejo de la formulación de las preguntas. La forma de recolección de la información por medio de las encuestas, fue llevada a cabo de manera personal para una real y mejor contribución de los encuestados.

La entrevista se hizo de manera estructurado mediante preguntas cerradas.

La revisión bibliográfica, también conocida con el nombre de revisión documental según Hernández-Sampieri, Fernández-Collado, y Baptista-Lucio (2006), consiste:

Detectar, obtener y consultar la bibliografía y otros materiales que pueden ser útiles para los propósitos del estudio, de donde se debe extraer y recopilar la información relevante y necesaria a la investigación. Esta revisión debe ser selectiva, puesto que cada año se publican en diversas partes del mundo miles de artículos de revistas, periódicos, libros y otra clase de materiales en las áreas del conocimiento (p. 23).

Por lo tanto, la revisión documental, se hizo en la unidad de transporte de la gobernación, que a partir del 2010 asume las otorgaciones de tarjetas de operaciones.

Los datos analizados, fueron obtenidos por medio de la aplicación de la encuesta a los pasajeros que viajan a las provincias del departamento de Potosí, para obtener las inquietudes de los viajeros sobre el transporte como son los servicios y si tiene conocimientos de una ley departamental.

\section{RESULTADO Y DISCUSIÓN}

El análisis de los resultados de encuestas relacionados con la variable cumplimiento, demostró que $71 \%$ de los sujetos encuestados correspondieron al sexo masculino y $29 \%$ al sexo femenino; $62 \%$ tenían edades comprendidas entre 18 y 
36 años; además el 51\% y la inmensa mayoría igual al 98\%, estuvo entre muy desacuerdo en el transporte interprovincial que no hay una ley departamental en transporte queregule los servicios, control y la fiscalización.

De esta forma se muestra a través de tablas los resultados obtenidos de acuerdo a las dimensiones desarrolladas pertinente al objeto central de la investigación.

\section{Clase de vehículo para viajar al interior del departamento de Potosí}

En la tabla 1 se muestran los resultados arrojados por los encuestados donde el $46,7 \%$ del total tiene preferencia para sus viajes el uso de (expresos), esto porque son más rápidos, cómodos, y son de puerta a puerta manifestaba un entrevistado. Por otro lado el $35,5 \%$ lo hace en flota, pues es un servicio, antiguo y es más cómodo, que los otros, pues brindan mayor seguridad y estabilidad afirman. Finalmente el $17,7 \%$, manifiesta que lo hace en Surubís, puesto que lo hacen por costumbre.

Tabla 1. Clase de vehículo

\begin{tabular}{lcc}
\hline ALTERNATIVAS & FRECUENCIA & $\%$ \\
\hline Flota & 16 & 35,5 \\
Surubí & 8 & 17,8 \\
Expreso & 21 & 46,7 \\
\hline TOTAL & $\mathbf{4 5}$ & $\mathbf{1 0 0 \%}$ \\
\hline
\end{tabular}

De esta forma, se evidencia que el expreso es la clase de vehículo que usan frecuentemente las personas para trasladarse al interior de departamento de Potosí.

\section{Seguridad y calidad en los vehículos para viajar al interior del departamento de Potosí}

En la tabla 2 se muestra que el $46,7 \%$ del total de pasajeros manifestaron que ningún transporte brinda un servicio de calidad, porque, se olvidan de los pasajeros, los choferes no son amables y cobran diferentes tarifas, factores que sin lugar a duda desacreditan los servicios de transporte. El 33,3\% de la población encuestada, manifestó, que el servicio es regular por las mismas variables mencionadas. Y solo un $20 \%$ del total, expresó que el servicio es bueno y que se sienten cómodos con las atenciones que brindan sobre todo los buses, que hacen el recorrido interprovincial o interdepartamental. 
Tabla 2. Seguridad y calidad vehicular.

\begin{tabular}{lcc}
\hline ALTERNATIVA & FRECUENCIA & $\%$ \\
\hline $\mathbf{S i}$ & 9 & 20 \\
No & 21 & 46,7 \\
Regular & 15 & 33,3 \\
\hline TOTAL & $\mathbf{4 5}$ & $\mathbf{1 0 0 \%}$ \\
\hline
\end{tabular}

\section{Optimización en la seguridad brindada a los pasajeros}

En la tabla 3, se muestran datos que corroboran los resultados referentes a la optimización de la seguridad brindada, así el, 46,6\% manifestó que el servicio brindado es regular, el $27,8 \%$, expresó que no se sienten seguros cuando hacen uso de un medio de transporte y solo el $25,6 \%$ afirmo que sí, hay seguridad cuando realizan viajes a provincias u otros departamentos. Por lo tanto, se determinó que sí hay falla en la seguridad brindada por medio del transporte público.

Tabla 3. Optimización en la seguridad.

\begin{tabular}{lcc}
\hline ALTERNATIVA & FRECUENCIA & $\%$ \\
\hline Si & 12 & 25,6 \\
No & 13 & 27,8 \\
Regular & 21 & 46,6 \\
\hline TOTAL & $\mathbf{4 5}$ & $\mathbf{1 0 0 \%}$
\end{tabular}

\section{Existe control y fiscalización antes, durante y después del viaje efectuado por funcionarios de transito}

En la tabla 4 se muestra, que el $60 \%$ manifestó que no hay control ni fiscalización por parte de las autoridades competentes, el 33,4\%, desconocían que existía un control por parte de funcionarios de tránsito. Y solo el 6,6\% del total argumento que sí, contaban con autoridades que se encargaban de ejercer control en las salidas de los buses o taxis, más propiamente en las trancas.

Tabla 4. Control y fiscalización por funcionarios de transito

\begin{tabular}{lcc}
\hline ALTERNATIVA & FRECUENCIA & $\%$ \\
\hline Si & 3 & 6,6 \\
No & 27 & 60 \\
Desconozco & 15 & 33,4 \\
\hline TOTAL & $\mathbf{4 5}$ & $\mathbf{1 0 0 \%}$ \\
\hline
\end{tabular}




\section{Reforma de la Ley Departamental del Servicio de Transporte interprovincial en el Departamento de Potosí}

Los datos que se muestran en la tabla 5 confirman y dan respuesta a la investigación, pues el $71,1 \%$ de encuestados expresaron que si era necesario regular con una Ley Departamental, el servicio de transporte interprovincial en el Departamento, en función de mejorar la calidad, servicio, seguridad acompañado del control y fiscalización por autoridades competentes. El 15,6\% desconoce las normativas vigentes y la necesidad de contar con una de ellas y el 13,3\% expresa que no sería bueno, puesto que sería otro motivo para que se les solicite dinero ilegalmente.

Tabla 5. Modificación de la Ley Departamental, el Servicio de Transporte Interprovincial.

\begin{tabular}{lcc}
\hline ALTERNATIVA & FRECUENCIA & PORCENTAJE \\
\hline Si & 32 & 71,1 \\
No & 6 & 13,3 \\
Desconozco & 7 & 15,6 \\
\hline TOTAL & $\mathbf{4 5}$ & $\mathbf{1 0 0 \%}$ \\
\hline
\end{tabular}

De esta manera, al analizar los resultados se determinó la ausencia de una ley en el departamento, por lo que es necesario contar con una normativa para un control sobre los servicios que dan los transportistas interprovinciales cuidando la calidad de un buen servicio para todos los usuarios que hacen uso de los servicios del transporte y así las autoridades competentes tomen carta en los problemas

\section{Propuesta de ley Departamental para la Otorgación de Tarjetas de Operaciones del Transporte Interprovincial en el Departamento de Potosí}

Objetivo General. Controlar y fiscalizar al transporte Interprovincial, con alcance de nivel Departamental para proteger sus derechos de los usuarios del servicio del transporte, en marco de los mecanismos de la ley que faculta.

\section{Objetivos específicos}

- Desarrollar acciones de capacitación a las autoridades pertinentes acerca del mecanismo de la ley otorgación de tarjetas de operaciones.

- Dar atención a las cosas que requieran la participación de un mediador cuando se encuentren en conflictos un operador o transportador, persona natural o jurídica que presta servicios del transporte. 
- Socializar la propuesta de ley de las tarjetas de operaciones en el Departamento de Potosí en el interior de los sindicatos de transportista, federación, mediante de charlas.

\begin{abstract}
Alcance
La ley tiene alcance de aplicación Departamental y provincial que regulara al transporte interprovincial que garantizara un servicio de calidad y seguridad a todoel usuario del servicio en el respeto de la población, y ejercer control y fiscalizacióna las funciones del servicio transporte
\end{abstract}

\title{
Justificación de la propuesta
}

En el marco de la tendencia actual, de la solución a la regulación del transporte en el Departamento de Potosí, es necesario que el Gobierno Autónomo, regule de manera legal, como la ley control y fiscalización de las tarjetas de operaciones, de esta manera se evitara las irregularidades en el transporte interprovincial y un servicio sea eficiente y de calidad.

Frente a las irregularidades en el servicio interprovincial, en el ámbito social y de usuario se encuentran desprotegidos de deben tomar medidas de hecho, que afectan al desarrollo normal de actividades de la población en general al uso del servicio que realiza al trasladarle a al provincial por ilegalidad del servicio.

Es necesario que a partir de la investigación, las autoridades reflexionen en pos de establecer garantías y principios de seguridad al transporte como existe en otros Departamentos que promueven políticas por un desarrollo del buen transporte a todas las provincias.

\section{Fundamentos jurídicos}

La Constitución Política como norma suprema, establece la distribución de competencias exclusivas de las Autonomías Departamentales art. 297, parágrafo I numeral 2, y son competencias de los gobiernos departamentales autónomos en su jurisdicción art. 300 parágrafo I numeral 9 son competencias exclusivas delos Gobiernos Departamentales.

Ley general de Transporte art. 17, emitir políticas regular, fiscalizar y control del sistema de transporte, el art. 21, ejercer competencias de transporte de alcance interprovincial, el art 25, en el ámbito de su jurisdicción y le corresponderá planificary regular, el art. 27, en el ámbito y materia de su competencia reglamentos, normasde carácter regulatorio, el art. 31, parágrafo II, otorgar permisos y autorizaciones, disposición transitoria primera de la ley 165, aplicaran normativas coordinación y regularan al problema de transporte. 
La ley N. a 031 marco de Autonomía y descentralización establece el art. 10, los aspectos regulan los aspectos inherentes a la autonomía, que encuentra en la Constitución política del Estado, en el art. 96 parágrafo III de acuerdo a la competencia exclusiva del numeral 9, parágrafo I del art 300, de la Constitución Política, los gobiernos departamentales tienen las competencias de ejercer control y fiscalización en los servicios del transporte interprovincial.

\section{Legislación comparada}

Ley Orgánica de Gobiernos Regionales Ley No 27867. Los Gobiernos Regionales tiene en materia competencia normativa de gestión y fiscalización, aprobaran nomas especificas en materia de transporte autorizar, supervisar y controlar la prestación de servicios de transporte interprovincial dentro el ámbito regional en coordinación con el gobierno regional.

Ley 10/2015, de 24 de marzo, por lo que establece el sistema competencial en el transporte urbano e interurbano de la región de Murcia, planificar, ordenar, gestionary regular el transporte, control, inspección y sanción, en su caso de los servicios de públicos.

Jurisprudencia administrativa, Disposición declarada valida. Consejo de Estado, Sección Primera, Sentencia del 25 de Julio de 2002, Radicación N 11001-03-24-0002001-01(6934), CP Olga Inés Navarrete.

La tarjeta de operación es el documento único que autoriza a un vehículo automotor para prestar el servicio público de transporte de pasajeros bajo la responsabilidad de una empresa de transporte, de acuerdo con los servicios autorizados.

Jurisprudencia: Corte Constitucional Sentencia C-006 de 1999.

\section{La naturaleza del transporte y los distintos fundamentos constitucionales de la regulación estatal en este campo}

Con la ley de transporte Departamental, se regulará el control y la fiscalización a las tarjetas de operaciones interprovincial, lo que otorgara seguridad jurídica y normativa partir de aplicación, orientando a brindar calidad en el servicio, además de las rutas, horarios y el parque automotor deberá contar con un control técnico actualizado.

El Proyecto de la Ley Departamental, tiene por objeto; regular y establecer las normas y procedimiento legales, administrativas y técnicas emergentes del Servicio Público del Transporte Automotor, Terrestre, Interprovincial y tiene por objeto la regulación del uso de las Tarjetas de Operaciones en las carreteras y caminos en la jurisdicción de Potosí; estableciendo y velando por el interés y la seguridad d la sociedad en su conjunto. 


\section{LEY DEPARTAMENTAL DE 30 DE NOVIEMBRE DE 2015JUAN CARLOS CEJAS UGARTE}

\section{GOBERNADOR DEL DEPARTAMENTO AUTÓNOMO DE POTOSÍ}

\section{“LEY DE REGULACIÓN DE TARJETAS DE OPERACIONES PARA EL TRANSPORTE TERRESTRE INTERPROVINCIAL DE PASAJEROS"}

La Ley de regulación de tarjetas de operaciones para el transporte terrestre interprovincial, está estructurado de la siguiente manera.

Capítulo I. Se muestra las dispersiones generales, distribuidos en 5 artículos (Potestad y competencia; objeto; objetivos específicos; ámbito de aplicación y definiciones.

Capitulo II. Tarjetas de operaciones. Conformado por 5 artículos (Tarjetas de operaciones; obligación de portarla; retención de la Tarjeta de operaciones; obligación de gestionarla y vigencia.

Capítulo III. El servicio del transporte. Incluye 5 artículos (Servicios; integración del transporte; y habilitación del transporte).

Capítulo IV. Competencia del gobierno Autónomo Departamental. Conformado por 3 artículo (Competencia del gobierno Autónomo Departamental; de la autoridad compétete del nivel Departamental y atribuciones).

Capitulo V. Funciones de la autoridad competente. Conformado por un artículo donde se desglosa las funciones de las autoridades.

Capítulo VI. Régimen de fiscalización de transporte terrestre. Se desarrollan 3 artículos (Competencia exclusiva de la fiscalización; elementos orientados de la fiscalización y alcance de la fiscalización).

Capítulo VII. Requerimientos administrativos. Conformado por 2 artículos (Responsabilidad de la Asamblea Legislativa Departamental de Potosí y Responsabilidad del Gobernador o Gobernadora del Departamental de Potosí).

Capitulo VIII. Requerimientos técnicos transporte de pasajeros. Está conformado por 2 artículos (Registro y categorización).

Capitulo IX. Régimen de sanciones. Conformado por más de 6 artículos (de las sanciones; multas; suspensión; inhabilitación; Sanciones a los Propietarios, Operadores o Conductores de Vehículos; reincidencia; otros).

Capitulo $\mathbf{X}$. Procedimiento administrativo para el régimen de sanciones. Conformado por 4 artículos (infracción, pruebas, secretaria, y resolución),

Capitulo XI. Seguros. Conformado por 6 artículos (Vigencia de los seguros; del seguro de los trabajadores; De la Obligatoriedad de los Seguros; Pérdida, Hurto o Destrucción total de un Vehículo). 


\section{CONCLUSIONES}

Tomando como base los resultados de la investigación se logró determinar que existe un vacío en la regulación de las tarjetas de operación del servicio de transporte interprovincial, por lo que se planteó la propuesta de una Ley Departamental que se encargue de regular, garantizar el control y fiscalización de las tarjetas de operaciones en el Departamento de Potosí, ya que, el Gobierno Autónomo Departamental de Potosí carece de una ley en transporte y de un plan que permita controlar al transporte interprovincial, para realizar un control y para determinar las condiciones de seguridad quedeben tener los vehículos para el transporte seguro de los, pasajeros que se encuentran imposibilitados para verificar el cumplimento de la normas de circulación y seguridad, necesaria para una prestación de un servicio, eficiente y confiable al pasajero.

Dando de esta manera, el cumplimiento del objetivo central de la investigación, logrando así, un mejor servicio al usuario, a través de la calidad del servicio, la seguridad y prevención al transporte.

\section{REFERENCIAS}

Bolivia. Decreto Supremo No 659, 6 de octubre de 2010

Constitución Política del Estado plurinacional de Bolivia Aprobada el 25 de Enero del 2009 Promulgada el 7 de Febrero del 2009

Fernández, J. (2002). Servicios públicos municipales, 1a. ed.; Ed. Porrúa México, Universidad Autónoma de México, México

Hawks, E. (1946). Historia del Transporte, Editorial juventud, Barcelona,

Hernández-Sampieri, R., Fernández-Collado, C., \& Baptista-Lucio, P. (2006). Análisis de los datos cuantitativos. Metodología de la investigación, 407-499.

Informe de Gestión 2006. Documento .Maracaibo. Venezuela.

Instituto Municipal de Transporte Urbano de la Ciudad de Maracaibo (IMTCUMA)

Lane, R., y TJ Powell. (1976). Planificación analítica del transporte. Instituto de Estudios. Administración Local. Madrid, España.

Ley General del Transporte Ley N¹65 del 16 de Agosto de 2011

Ley Marco de Autonomías y Descentralización Andrés Ibáñez ley n031 19 de juliode 2010
Lewis, R., y Booms, B. (1983). They marketing aspect on of service quality, en Berry, LL, Shotok, G.L. y Upah.G.P. (Eds.). Emerojing perpectives on services Marketing A.M.A. Chicago: 99-10)

Núñez, L (2001). Reflexiones para una teoría servil.

http://hogF.org/foranica/política/opinión, html.15nobiembre2001

Ocaña, R. (2003). Análisis y Evaluación del Programa Nacional de Transporte Urbano en Venezuela (1991-2000) Trabajo de Ascenso. Universidad Simón Bolívar. Caracas.

Osorio, M. (2002). Diccionario de Ciencias jurídicas Políticas y Sociales. Editorial Heliasta, Argentina

Rubio, MJ., y Varas. (1979). El Análisis de la Realidad Social en la Intervención Social, Madrid, CCS, PP.

Taylor, SJ., y Bogdan (1972). Introducción a los Métodos Cualitativos en la Investigación. La Búsqueda de lo Significado. Ed. Paidós. España

Valero, J. (1970). Transporte Urbano Madrid .N de Referencia. De la librería 008475DZA 